\section{Nierenversagen: Steine als Vorboten}

Erwachsene mit mindestens einem symptomatischen Nierenstein entwickeln innerhalb von elf Jahren etwa doppelt so häufig ein terminales Nierenversagen wie Patienten ohne Steine, wie eine aktuelle Studie ergab.

In einer kanadischen Studie wurden die Daten von fast zwei Millionen Krankenversicherten ausgewertet. Im Zeitraum von 1997 und 2009 war bei 23.076 Studienteilnehmern $(0,8 \%)$ mindestens einmal eine Nephrolithiasis diagnostiziert worden. 68.525 Personen ( $4 \%$ ) entwickelten eine chronische Niereninsuffizienz im Stadium 3b-5 (definiert durch eine geschätzte glomeruläre Filtrationsrate $\left.<45 \mathrm{ml} / \mathrm{min} / 1,73 \mathrm{~m}^{2}\right)$. Bei 5.333 Personen $(0,2 \%)$ wurde eine Nierenersatztherapie notwendig. $\mathrm{Zu}$ einer anhaltenden Verdopplung des anfänglichen Serumkreatinins kam es bei 6.581 Patienten $(0,3 \%)$.

Unter Berücksichtigung von Alter, Geschlecht und Komorbiditäten hatten Steinpatienten im Vergleich zu steinfreien Personen ein 2,16-fach erhöhtes Risiko für ein terminales Nierenversagen. Auch die Wahrscheinlichkeiten für eine chronische Nierenkrankheit im Stadium 3b-5 sowie für eine Verdopplung des Serumkreatinins waren signifikant erhöht, und zwar um $74 \%$ beziehungsweise $94 \%$. Besonders deutlich zeigte sich der Zusammenhang bei Frauen und Personen unter 50 Jahren. Patienten mit mehreren Episoden von Nierensteinen waren ebenfalls besonders gefährdet. Allerdings war die absolute Risikosteigerung relativ klein: Pro einer Million Personentage wurden 2,48 vs. 0,52 Fälle einer terminalen Niereninsuffizienz und 61,47 vs. 25,5 Fälle von chronischer Niereninsuffizienz ab Stadium $3 b$ registriert.

Die Ursachen für die fortschreitende Nierenschädigung bei Patienten mit kalziumhaltigen Nierensteinen sind wahr- scheinlich multifaktoriell. Zum einen kann es sich um direkte Folgen der Kalzifizierung im Niereninterstitium und in den Lumen der Tubuli handeln. Des Weiteren könnten durch die Steine verursachte Harnwegsobstruktionen die Nierenfunktion beeinträchtigen. Denkbar ist auch, dass durch die operative oder perkutane Beseitigung der Steine Schäden gesetzt werden. Eher selten dürften monogenetische Erkrankungen wie eine primäre Hyperoxalurie oder eine Cysteinurie dazu beitragen.

Fazit: Nach den Ergebnissen dieser Studie sollten Patienten mit Nierensteinen in der Anamnese als Patienten mit einem erhöhten Risiko für ein Nierenversagen angesehen werden. Dies gilt vor allem für jüngere Frauen und für Patienten mit mehreren Steinepisoden.

Dr. Beate Schumacher

Alexander RT et al. Kidney stones and kidney function loss: a cohort study. BMJ 2012; 345: e5287

\section{Weniger Prostatakarzinome bei Statinanwendern}

\section{Durch Statine können Männer nicht nur ihren Cholesterinspiegel, sondern möglicherweise auch ihr Prostatakarzinomrisiko senken. Das legt eine Metaanalyse nahe, in der alle einschlägigen Beobachtungsstudien aus den Jahren 1993 bis 2011 ausgewertet wurden.}

$\mathrm{D}$ ass Statine in der Lage sind, das Wachstum von Prostatakarzinomen zu unterdrücken, ist im Tierexperiment und in klinischen Studien gezeigt worden. Die Auswirkungen der Cholesterinsenker auf das Prostatakrebsrisiko sind jedoch strittig. In den jüngsten Metaanalysen aus dem Jahr 2008 wurde kein signifikanter Zusammenhang festgestellt. Da seitdem eine Vielzahl weiterer Studien publiziert wurde, haben indische und britische Mediziner nun erneut Bilanz gezogen. Ihre Metaanalyse fußt auf 27 Studien, 15 Kohortenstudien und zwölf Fall-KontrollStudien aus den Jahren 1993 bis 2011. An den Studien waren fast 1,9 Millionen Männer beteiligt, die Beobachtungszeit lag zwischen zwei und 17 Jahren, bei 56.847 Männern wurde Prostatakrebs entdeckt.
Die Ergebnisse der einzelnen Studien waren sehr heterogen, eine Verminderung des Krebsrisikos unter Statinen wurde in elf Studien beobachtet. Insgesamt ergab sich für Statinpatienten eine um $7 \%$ niedrigere Prostatakarzinomrate (RR 0,93; $95 \%-\mathrm{KI} 0,87-0,99 ; \mathrm{p}=0,030)$. Fortgeschrittene Prostatakarzinome, meistens definiert als Tumoren der Stadien T3/T4, wurden bei ihnen sogar um $20 \%$ seltener diagnostiziert (RR 0,80; $95 \%$-CI $0,70-$ 0,90; p < 0,001). Kein Rückgang der Krebshäufigkeit zeigte sich allerdings in den Studien, in denen die Langzeitanwendung von Statinen über mehr als fünf Jahre untersucht wurde.

Schwächen in der Metaanalyse sehen die Autoren in dem Umstand, dass bekannte Risikofaktoren für Prostatakrebs wie Übergewicht und ein ungesunder
Lebensstil nur in elf Studien berücksichtigt wurden. Angaben zur Teilnahme an einem PSA-Screening und zu PSA-Werten enthielten sogar nur sechs Studien. Dabei gibt es Hinweise, dass sich Statinpatienten häufiger an Reihenuntersuchungen beteiligen, ein Umstand, der eine frühe Tumordiagnose erleichtert. Andererseits senkt eine Statintherapie den PSA-Spiegel und könnte dadurch die Diagnosestellung verzögern.

Fazit: Die Ergebnisse der Metaanalyse legen ein vermindertes Prostatakarzinomrisiko bei Statinanwendern nahe. Als Ursache dafür kommen eine Vielzahl bekannter Statinwirkungen infrage, etwa die Hemmung von Entzündung, Angiogenense, Zellproliferation und Zellmigration sowie die selektive Förderung der Apoptose von Tumorzellen.

Dr. Beate Schumacher

Bansal D et al. Statin Use and Risk of Prostate Cancer: A Meta-Analysis of Observational Studies. PLoS One 2012; 7: e46691 